\title{
Monism: the Islands of Plurality
}

\author{
Sam Baron \\ University of Western Australia \\ Jonathan Tallant \\ University of Nottingham
}

\begin{abstract}
Priority monism (hereafter, 'monism') is the view that there exists one fundamental entity-the world—and that all other objects that exist (a set of objects typically taken to include tables, chairs, and the whole menagerie of everyday items) are merely derivative. Jonathan Schaffer has defended monism in its current guise, across a range of papers. Each paper looks to add something to the monistic picture of the world. In this paper we argue that monism-as Schaffer describes it-is false. To do so we develop an island universe' argument against Schaffer's monistic theory.
\end{abstract}

\section{Introducing Monism}

At the outset, we spell out Schaffer's monism in more detail. In so doing, we consider the view as he presents it across three papers (Schaffer (2009, 2010a, 2013)); we defer discussion of the fourth paper (Schaffer (2010b)) until a little later on.

As we understand it, monism is the thesis that 'the world' or 'the cosmos' is the single fundamental object upon which all other objects (objects that are parts of this fundament) depend for their existence. In Schaffer's words:

The monist holds that the whole is prior to its parts, and thus views the cosmos as fundamental, with metaphysical explanation dangling downward from the One. (2010a: 31)

As Schaffer makes clear (2010a: 38), he is interested only in concrete objects. Accordingly, monism is the view according to which there exists a single concrete object that is (metaphysically) basic and that is the cosmos. Of course, to say that one object is basic allows that there exist non-basic objects. According to Schaffer, in addition to basic objects, there exist derivative (or, non-fundamental) objects. These objects are parts of the monistic whole, and include things like tables, chairs and other familiar macroscopic objects (cf. (2010a: 33)).

There are, however, still questions as to how we understand the relationship between concrete objects, the monistic substance, and the spacetime in which they are found. Here we turn to Schaffer (2009) who presents us with two options. First, objects (both basic and derivative) may be said to exist in addition to the spacetime in which they are embedded. Second, objects (both basic and derivative) may be identified with various portions of spacetime. Schaffer (2009: 133) takes monism to be (and defends it as the thesis that) the monistic substance is to be identified with spacetime. To be clear there are really two views here: first 'priority monism' according to which the whole cosmos is prior to its proper parts and, second, 'monistic substantivalism' according to which material objects are not distinct from regions of substantival spacetime. Schaffer endorses both views, though one need not. The views are, in principle at least, separable. In this paper, however, we are primarily interested in Schaffer's monism and so will continue to assume that priority monism and monistic substantivalism go together. As such, the monistic substance is a spacetime and every part of the spacetime is to be understood as a concrete object.

There are four distinct types of argument that Schaffer deploys to motivate Monism, and that we will focus upon here: gunk, integration, physics and in a further paper-his (2010b)—an argument from truthmakers. We compress the arguments somewhat, in the interests of space. However, all of the arguments are relevant to the later dialectic and since we do not assume that the reader is already familiar with the totality of Schaffer's corpus, we include these brief discussions here. We do not defend Schaffer's arguments. Our concern in this paper is whether or not, even on its own terms, Schaffer's monism so understood is a defensible view.

\subsection{Gunk.}


As Schaffer has it, monism is opposed by pluralism; the view that more than one object is basic. But we have reason to think that pluralism is false, claims Schaffer, as shown by the gunk argument. To begin, note that ' $[\mathrm{t}] \mathrm{he}$ pluralist holds that the parts are prior to their whole, and thus tends to consider particles fundamental, with metaphysical explanation snaking upward from the many.' (2010a: 31)

The gunk-hypothesis is the hypothesis that concrete objects are such that every proper part of a concrete object itself has a proper part. The gunk-hypothesis may be actually true; it may not be. But, claims Schaffer, we have good reason to think that gunk is metaphysically possible 1 and a 'gunk world' (a world at which every concrete object is 'gunky') would have no 'bottom level'- there would be no ultimate smallest constituent at such a gunk-world as whatever object one identifies will, itself, have proper parts. At such a world, then, there is no 'bottom layer' for metaphysical explanation to 'snake upward from'. More starkly: 'nothing is basic at gunky worlds. There would be no ultimate ground. Being would be infinitely deferred, never achieved' (Schaffer, 2010a: 62). If being is never achieved, then nothing in fact exists. But since things do exist, so this picture must be wrong. ${ }^{2}$

Now, monism and pluralism are such that they are, if true, then necessarily true (2010a: 62). If gunk is possible, then we must reject pluralism. And so since pluralism and monism are exhaustive (cf. 2010a: 33), so monism is both necessarily and actually true. Monism faces no problems with gunk. The whole-the cosmos-is simply infinitely divisible, with each division less metaphysically basic than the last. To lean on the same metaphor: metaphysical explanation snakes downward from the one. Thus, if we assume that some concrete object is metaphysically basic, we have an argument for monism. ${ }^{3}$

\subsection{Integration and Action}

Schaffer's second argument for monism concerns the notion of integration. A preliminary version of the argument is an argument from common sense. To articulate the argument, Schaffer (2010a: 47) begins by noting a distinction between two kinds of wholes: integrated, unified wholes, and mere aggregates. A pile of stones nicely illustrates a mere aggregate. In a (mere) pile, there is no sense of the whole being integrated in any way. Rather, there is simply an un-integrated collection, a sum. In contrast, there are cases where wholes do seem to be integrated. Consider, for example, a body. The parts of a body (the organs, bones, etc.) do not seem to be arbitrarily piled together as the parts are in a sum. Rather, the parts of a body are tightly integrated so-as to ensure they can perform various tasks. Drawing upon this, Schaffer (2010a: 49) claims that common sense also recognizes a distinction in terms of priority. In cases of integrated wholes, Schaffer argues that it is natural to suppose that the parts depend upon the whole (to develop the example, that organs, bones, etc., depend for their existence upon the body that houses them). In contrast, in cases of mere aggregates, Schaffer argues that it is natural to suppose that the whole depends upon the parts. Since the cosmos that we inhabit exhibits integration, so we should think that the cosmos is prior to its parts.

In subsequent work, Schaffer (2013) develops a version of the argument from integration (that Schaffer here calls Spinozan Monism) that does not rely on common sense. The argument, briefly stated, is this:

(1) Leibnizian Substance: Something is a substance if and only if it evolves by the fundamental laws.

(2) Russellian Laws: The cosmos is the one and only thing that evolves by the fundamental laws.

(3) Spinozan Monism: The cosmos is the one and only substance (from 1 and 2).

(Schaffer 2013, p. 67)

Crucial to this argument is the idea of 'evolving by the fundamental laws'. By 'fundamental law' Schaffer has in mind the laws that appear in our most fundamental physical theories. Here's what it means to evolve by the laws:

Evolving Something evolves by the fundamental laws if and only if, for any given time, its prediction at that time matches its behavior.

\footnotetext{
${ }^{1}$ Cf. Schaffer (2010a: section 2.4); Schaffer (2003).

2 Cf. Tallant (2013: 430).

3 At least, so says Schaffer. See Tallant (2013) for reasons to be less confident on this score.
} 
Where 'behaviour' and 'prediction' are parsed in terms of a 'state' as follows:

State The state of a thing at a time is its fundamental intrinsic character at that time.

Prediction The prediction for a state is the temporal evolution that the fundamental laws output on the basis of the state.

Behavior The behavior of a thing is the temporal evolution it actually displays.

Now, note that Schaffer defines a substance in terms of integration, as follows:

Substance Something is a substance if and only if it is a fundamental and integrated thing.

Leibnizian Substance then forges a link between substance and evolution by law via the concept of integration. Here's Schaffer:

Leibnizian Substance draws on the idea of substances as integrated, and can be understood as providing a nomic test for natural unity. To evolve by the fundamental laws is to act in an integrated way, forming an internally comprehensible and self-contained system [...] The natural unity of a thing is displayed in its dynamics. In a slogan: to be one is to act as one. (Schaffer 2013: 72)

Integration, then, has a decidedly nomic flavor: two entities are appropriately integrated to the extent that they 'act as one' in accordance with the fundamental laws. The notion of integration so understood plays a central role in Schaffer's case for Spinozian Monism from Leibnizian Substance and Russellian Laws. In particular, it is used to defeat the chief rival, Leibnizian Substance, Plural:

Leibnizian Substance, Plural Some things are substances if and only if they co-evolve by the fundamental laws.

The central objection against this pluralist thesis, as stated, is that it does not preserve the link between integration and evolution via the laws. As Schaffer puts the point:

But Leibnizian Substance, Plural does not fit the idea of substances as integrated [...] Indeed, it seems to me that a plausible way to decide whether a given composite system is most fundamentally a single composite substance or a mere heap of various elements is to see whether the plurality acts as one. So if some things co-evolve by the fundamental laws, it seems to me that one ought to conclude, not that each of these things is an individual substance, but rather that their system is a composite substance, for that is what forms a natural unit with respect to the dynamics. (Schaffer 2013, p. 73).

Schaffer's argument for Spinozian Monism gives us a great deal to consider. For now, however, we wish to only draw out two points.

First, nomic integration is essential to substancehood: if an entity $E$ does not 'act as one'-where that means there is some fundamental law, or set of laws, by which $E$ evolves - then $E$ is not a substance. Thus, a mere heap, the evolution of which is not governed by a fundamental law (or set of laws), is not an integrated whole; it is not a substance. (Though, of course, it is a part of a substance by virtue of being a part of an entity (the world) whose evolution is so governed.) Conversely, if an entity $E$ does 'act as one', then that is sufficient for E's substancehood.

Second, a natural move that the monist might make against the pluralist is to reconstrue two entities that seemingly co-evolve, as one composite entity that evolves by a single set of fundamental laws. Thus, if we seemingly have a case of two entities, $E$ and $E^{*}$, and although it seems that $E$ and $E^{*}$ merely co-evolve, following distinct laws, $L$ 
and $L^{*}$, then though this may seem to favor pluralism, this need not prove decisive so long as one can tell a convincing story about the fusion of $E$ and $E^{*}$ evolving according to some fundamental law, $L_{F}$.

\subsection{The physics}

Schaffer offers two tranches of argument intended to favour monism based on current physics-specifically, the monistic thesis that the cosmos qua spacetime whole is prior to its parts. One tranche of arguments looks to identify various properties that appear to be emergent from quantum-mechanical interactions, and that seem to suggest monism. ${ }^{4}$ We will not pursue this argument in favour of monism any further.

The second tranche of argument is one that will occupy a little more of our attention. Schaffer claims that there are certain properties, exhibited by spacetime, that are properties of a spacetime itself rather than properties of mere regions of a spacetime. By way of example, consider topological and geometrical properties of a spacetime: 'topological notions such as disconnectedness (where a region is connected if and only if it is not the union of any two non-null separated regions), and geometrical notions such as distance (understood via the length of the shortest path between two points), make implicit reference to the whole manifold' (Schaffer, 2009: 135). Schaffer (2009: 136) then gives the following further quotation from Bricker (1993: 228):

Manifold structure is in part topological structure, and topological structure, it is easy to see, is irreducibly global. Consider a two-dimensional Euclidean plane (and the surface of) an infinite cylinder. They are locally indistinguishable: each consists of continuum-many points that are locally Euclidean. But the plane and the cylinder differ topologically. For example, the plane, but not the cylinder, is simply connected: all closed paths can be continually contracted to a point.

Schaffer's claim is that because the topological and geometrical features at issue are global, the parts of spacetime that possess those features must do so in virtue of being embedded within spacetime itself. Accordingly, the parts of spacetime have the features that they do because they are grounded in spacetime and not vice versa. It follows that the parts of spacetime are posterior to spacetime itself, which is fundamental.

\subsection{Truthmaker concerns}

Truthmaker maximalists claim that every truth has a truthmaker (non-maximalists claim that only some truths have truthmakers, and attempt to provide a non-ad-hoc restriction on which truths require truthmakers; ${ }^{5}$ for instance, see Simons (2008) and Tallant (2010)).

Roughly, this amounts to the thesis that a proposition-any proposition-depends for its truth upon the existence of some entity or other. Maximalists then face a problem: there is no obvious entity, $E$, such that $<$ there are no dragons $>$ (or any other so-called 'negative existential proposition') depends for its truth upon E. After all, any of the contingent objects that in fact exist are compossible with dragons and, as such, cannot be regarded as that 'in virtue of which it is true that <there are no dragons $>$ - else it would be possible for < there are no dragons $>$ to be true and for dragons to exist.

The candidate entities often appealed to in order to do the work necessary are 'negative'. So, for instance, some are minded to posit as truthmakers negative properties (the truthmaker for <there are no dragons $>$ is the world's instantiating the property not containing dragons) or other posits with a negative nature (a totality relation (Armstrong (2004)); negative facts (Barker and Jago (2012)) and polarities (Priest (2001)) have all been called upon). Nonetheless, there remains a suspicion that these negative entities are-in some sense or otherobjectionable. ${ }^{6}$

Schaffer (2010b) claims that there is a concrete object whose existence is not compossible with the existence of dragons, that is not negative, and, as such, can be said to be that in virtue of which < there are no dragons $>$ is true.

\footnotetext{
${ }^{4}$ We say 'seem' since, following Morganti (2009) we do not find these arguments convincing.

${ }^{5}$ We deliberately set-up the problem here in a way that should be amenable to a Schafferian analysis, treating the truthmaker relation as 'in virtue of rather than 'necessitation' as is more common.

${ }^{6}$ Those who push the line that negative ontology is objectionable include Dodd (2007) and Molnar (2000). Whilst we're also mindful of the fact that there is no clear agreement in the literature as to when a posit is negative-and that there is some scepticism about the term 'negative' in this context—-we're prepared to allow Schaffer that his argument goes through.
} 
That concrete object is the world — the one fundamental substance. Once we fix the nature of the world-once we fix the nature of the fundament-we fix which truths there are. Here is Schaffer's (2010b: 321) reasoning:

In general, any expansion (any more to the world) requires a different unique fundament - if the worlds in question did not differ, then they would be indiscernible, rather than contracted and expanded. So $<$ there are no dragons $>$ is true at actuality, in virtue of actuality's being the unique actual fundament. Once actuality is listed as the one fundamental entity, ground for the absence of dragons is assured.

Whether or not it is desirable to locate truthmakers for negative existential propositions is something over which we are uncertain. However, assuming that this is so, we are prepared to allow Schaffer that, as he puts it (2010b: 321), if monism can solve the problem of negative existentials whilst not committing us to negative entities, then this is 'some justification for the monistic thesis.'

\section{Islands of Plurality.}

We have in hand four arguments that collectively serve to motivate Schaffer's monism, and we have a reasonably clear handle upon the view itself. We now want to advance our argument against monism. The argument is a modal argument, based on the possibility of island universes.

First, though, a quick word about possible worlds: for Lewis (1986: 75-76) possible worlds are unified via their spatiotemporal relations (or analogically spatiotemporal relations). In what follows, we will adopt a more liberal approach to the relations that unify worlds. Following Bricker (1996), we allow that worlds may be unified by non-spatiotemporal relations. Which ones? Here's Bricker (1996: 230):

In the analysis of world, the family [of unifying relations] must contain natural external relations that are not spatiotemporal. But which ones? It would be arbitrary, I think, to include some while excluding others. I propose, then, that we take [the family of unifying relations] to contain all natural external relations: worlds are maximally externally unified regions of logical space; all and only worldmates are externally related.

As Bricker (1996: 229) argues, there are live scientific hypotheses about our world according to which the world is not spatiotemporal in nature. Some of these hypotheses arise in early work on quantum mechanics, as a way of making sense of phenomena such as wave-particle duality. However, more recent work in quantum gravity has seen an explosion of non-spatiotemporal accounts of reality. ${ }^{7}$ To take just one example, so-called timeless theories of quantum gravity such as the Machian interpretation of canonical quantum gravity developed by Barbour (1999) argue for the elimination of spacetime altogether. ${ }^{8}$ Accordingly, such worlds are not unified by spatiotemporal relations but, rather, are unified by laws governing a much broader configuration space. As with Bricker, we take it that a live scientific hypothesis about actuality should, at the very least, be a possibility, and thus that the notion of unification appropriate to worlds must be expanded beyond spatiotemporal connection. ${ }^{9}$ In order to prevent further conflicts with science, the most straightforward thing to do is let any external relations bind relata into a world, as Bricker does.

With these remarks in hand, we now turn to a discussion of island universes. Island universes have been discussed most extensively in the literature on possible worlds as, famously, they cause a potential worry for Lewis's (1986) reduction of modality. Following Bricker (2001)—though see, also, Bricker (1996)—we take it that an island universe is one in which there are two or more distinct spacetimes that are nonetheless members of the same universe. Such worlds as these, that Bricker (2001: 32-33) calls locally unified worlds, include 'separate parts'. The 'separate parts' belong to a single region of logical space-as opposed to a region of spacetime. There are two forms of the argument against monism based on island universes. The distinction

\footnotetext{
${ }^{7}$ To be sure, Lewis (1986: 75-76) allows that analogically spatiotemporal relations might unify. Some theories of quantum gravity, however, do not deploy even analogically spatiotemporal relations.

${ }^{8}$ See Baron and Miller (2015) for an overview of some timeless theories of quantum gravity.

${ }^{9}$ We note that Schaffer (2010a: 34-5) seems to agree with the claim that spatiotemporal relations are not essential to worldbuilding, offering instead part-hood as the relation required for a world. The view seems to be that $x$ exists at $W$ iff $x$ is a part of $W$.
} 
between the two arguments tracks a prior distinction that Bricker draws between two kinds of island universes: weak and strong. ${ }^{10}$

Weak Island Universes: $x$ is a weak island universe iff $x$ fails to be externally unified by any sparse spatiotemporal relation or by any causal, spatial or temporal relation (or any combination thereof).

Strong Island Universes: $x$ is a strong island universe iff $x$ fails to be externally unified by any sparse external relation.

Crucial, here, is the distinction between an internal and an external relation, which Bricker (2001: 31-32) draws as follows:

An internal relation is a relation that supervenes on the intrinsic natures of its relata. Having-the-samemass-as is an example of an internal relation, assuming the mass properties are intrinsic. An external relation is one that, although it fails to supervene on the intrinsic natures of its relata, does supervene on the intrinsic natures of its relata, and of the fusion of its relata.

Next: unification (Bricker 2001: 30-32). Two parts of a world $W, P_{1}$ and $P_{2}$ are weakly separated, just when $P_{1}$ and $P_{2}$ are non-overlapping and no part of $P_{1}$ stands in any sparse external spatiotemporal, causal, spatial or temporal relation (or any combination thereof) to any part of $P_{2}$. A world $W$ is therefore externally unified by a sparse spatiotemporal, causal, spatial or temporal relation (or any combination thereof), just when it is not the sum of two parts that are weakly separated. Two parts of a world $W, P_{1}$ and $P_{2}$ are strongly separated just when $P_{1}$ and $P_{2}$ are non-overlapping and no part of $P_{1}$ stands in any sparse external relation to $P_{2}$. A world $W$ is therefore externally unified by a sparse external relation when it is not the sum of two parts that are strongly separated.

In what follows, we will for the most part think in terms of disconnected spacetimes. Thus, a weak island universe par excellence, is a universe in which there are two or more spatiotemporal manifolds that are not externally unified by any sparse spatiotemporal, spatial, temporal or causal relation; whereas a strong island universe par excellence, is a universe in which there are two or more spatiotemporal manifolds that are not externally unified by an sparse external relation at all. Talking in terms of disconnected spacetimes is for ease of exposition only. Ultimately, worlds with disconnected spacetimes are but a species of island universe: any world that fails to be globally unified in either the weak or strong sense - even if it is a world in which there is no spacetime at all - is an example of an island universe. This will become important much later on, when we consider what it would take to rule against the possibility of island universes. For now, however, we set the matter to one side and press on.

We can now state our argument against Schaffer's particular brand of monism:

(1) Monism is the thesis that 'the world' is a single spacetime, each part of which is a concrete object, such that each part is dependent upon the spacetime.

(2) If true, monism is necessarily true.

(3) Island universes are possible.

(4) If island universes are possible, then monism is not necessarily true.

Given (2), (3) and (4) and modal equivalences:

(5) Monism is not true.

There is work to do. We have already established Schaffer's commitment to premises (1) and (2). So, first we'll look at reasons to think that there are weak island universes (2.1) then we'll take a look at reasons to think that there are strong island universes (2.2). This will yield two different interpretations of the argument just stated, with slightly differing commitments at premise (3): one that trades upon the possibility of weak island universes; one that trades upon the possibility of strong island universes. We will make these different arguments precise (2.3) by offering arguments for the possibility of both kinds of island universe. We will then defend premise (4). ${ }^{11}$ In $\$ 3$ we will consider attempts to undermine premise (3) - in both its forms. We argue that these attempts are not successful.

\footnotetext{
${ }^{10}$ See Bricker (2001: section 2.1) for a precise analysis of what it is to be an island universe.

11 More precisely: we will need to defend two slightly different versions of premise (4): one for each interpretation of premise (3).
} 


\subsection{Weak Island Universes}

Here's the argument for the possibility of weak island universes. As Bricker (2001: 35-36) notes (see also Weingard (1976)), there is a well-known solution to Einstein's field equations for general relativity in which there are two regions of spacetime $I_{1}$ and $I_{2}$ that are connected by a short-lived wormhole. The wormhole constitutes a spatiotemporal bridge between $I_{1}$ and $I_{2}$, and evolves spontaneously, growing to a maximum size before shrinking and then 'pinching off, collapsing the bridge between $I_{1}$ and $I_{2}$, at which point $I_{1}$ and $I_{2}$ are spatiotemporally disconnected.

This solution to the field equations does not yet establish the possibility of an island universe. However, this can be established by adding some degree of physical indeterminism into the picture. As Bricker (2001: 35) puts the point:

Thus far, we have only spatial, not spatiotemporal, isolation. But now consider physical indeterminism. Presumably, there was some non-zero objective chance that the "wormhole" would never have evolved, that is, some non-zero objective chance that the two regions would have been spatiotemporally isolated island universes. And whatever has a non-zero objective chance of happening is physically possible.

Bricker attributes the move here to an earlier paper by Bigelow and Pargetter (1987), but stops short of outright endorsing the argument, saying, "I don't really know whether our best physical theory allows general relativity and indeterminism to be combined in the way required for this argument" (Bricker 2001: 35).

In fact, general relativity and indeterminism can be combined in the manner imagined. At very small scales, namely the Planck scale, the topological structure of spacetime is subject to quantum fluctuations that can give rise, with some probability, to very small wormholes. Crowell (2005: 57-58) has recently provided a solution to the Wheeler-DeWitt equation that can be used to describe quantum wormholes according to which the resulting geometric picture is one involving a "quantum fluctuation that connects two spacetime cosmologies" via a wormhole that lasts for a very brief interval, at the order of around 10 Planck units of time. These two spacetime cosmologies, as Crowell makes clear, are distinct spacetimes, in so far as they are not spatiotemporally connected prior to, and following, the quantum fluctuation at issue.

The Crowell case is exactly the case that Bricker envisages: one in which two disconnected spacetimes are connected by a wormhole, where the connection event has an objective chance of not happening. This model seems to be physically possible. It follows, then, that there is a world with two disconnected spacetimes in which the required quantum fluctuation never happens. This will be a weak island universe. ${ }^{12}$

\subsection{Strong Island Universes}

Establishing the possibility of strong island universes is more involved. We will begin with an argument, once again, from Bricker. Bricker's argument for the possibility of strong island universes begins with the following principle:

Generalized Principle of Solitude for Particulars (GPSP): For any particular (thick, thinned-down, or thin), possibly, a duplicate of that particular exists all by itself.

Note that a thick particular, is, very roughly, an individual bundled with properties of various kinds. A thin particular is an individual stripped of its properties - a so-called 'bare' particular. The 'thinning down' of a particular is removal (via recombination) of more and more of that particular's properties to get down to its bare

\footnotetext{
${ }^{12}$ One might demur: surely it only makes sense to say that two spacetimes are connected to one another by a wormhole if there is a higher dimensional space in which the two spacetimes are situated, and through which the wormhole tunnels. This, however, is certainly not forced on us, at least not for any geometrical reason pertaining to general relativity that we can see. All we need to do is think of the wormhole as, itself, a piece of spacetime that bridges two distinct spacetime cosmologies. As a piece of spacetime itself, the wormhole does not need to be situated in a higher dimensional space any more than spacetime more generally needs to be. There should probably be some relation between the two spacetimes in virtue of which there is some objective chance that a wormhole might connect one spacetime to another. Bricker rules out any internal relation, since an internal relation would mean that for every duplicate of the two spacetimes, there is a nonzero chance that a wormhole might connect the duplicates, effectively ruling out the isolation of the spacetimes at issue within lonely worlds - worlds without island universes. So the relation should be an external one.
} 
nature (if it has one; if bare particularity is even coherent). (In what follows, we will talk of recombination in part through metaphor. Thus, we will talk of 'stripping away' or 'removing' particular properties or relations. We do not mean this talk literally. As is the norm in the literature, we use such metaphors to express the idea that the resultant worlds are possible given principles of recombination. $)^{13}$

Using GPSP it is relatively straightforward to show that strong island universes are possible. First, take a spacetime $M$ and use it to identify two individuals, $I_{1}$ and $I_{2}$. $I_{1}$ is made up of all of the objects $O_{1} \ldots O_{n}$ in a spacetime region $R$ in $M$ plus the spacetime relations between those objects; $I_{2}$ is made up of all of the objects $O^{*}{ }_{1} \ldots O_{2}^{*}$ in a spacetime region $\mathrm{R}^{*}$ in $M$ (where $\mathrm{R}$ is not identical to $\mathrm{R}^{*}$ ) plus the spacetime relations between those objects. In the interests of clarity, we will also specify that $I_{1}$ and $I_{2}$ do not overlap and are at some distance from one another. Now, and this is crucial, as we define things here, neither $I_{1}$ nor $I_{2}$ include the spacetime relations to each other.

Second, take the mereological sum, $S$, of $I_{1}$ and $I_{2}$. By GPSP we can 'strip away' the spacetime relations between $I_{1}$ and $I_{2}$, as well as any regions other than $I_{1}$ and $I_{2}$, to get to a world in which $S$ exists without spatiotemporal relations between $I_{1}$ and $I_{2}$. We can do this because GPSP tells us that, possibly, there is a duplicate of the sum $S$ that exists all by itself, where that means without any of the spatiotemporal relations that bind $I_{1}$ and $I_{2}$ together and without any further sparse external relations binding those regions together either. A world in which $S$ exists without sparse external relations connecting $I_{1}$ and $I_{2}$ would be a world in which there are two disconnected spacetimes and would qualify as a strong island universe.

\subsection{The Argument made precise.}

In $\$ 2$, we gave a brief statement of our argument.

(1) Monism is the thesis that 'the world' is a single spacetime, each part of which is a concrete object, such that each part is dependent upon the spacetime.

(2) If true, monism is necessarily true.

(3) Island Universes are possible.

(4) If Island Universes are possible, then monism is not necessarily true.

Given (1)-(4) and modal equivalences:

(5) Monism is not true.

We also noted that some precisification would be required in order to respect the distinction between weak and strong island universes. Precisifying the premises delivers:

(3*) Weak island universes are possible.

$\left(3^{* *}\right)$ Strong island universes are possible.

In turn this delivers the promised (see fn. 11) revision to premise (4):

(4*) If weak island universes are possible, then monism is not necessarily true.

(4**) If strong island universes are possible, then monism is not necessarily true.

Call the argument that deploys $\left(3^{*}\right)$ and $\left(4^{*}\right)$ the 'modest argument' against monism and the argument that deploys $\left(3^{* *}\right)$ and $\left(4^{* *}\right)$ the 'proud argument' against monism. We have already defended premises $\left(3^{*}\right)$ and $\left(3^{* *}\right)$. It remains to say something in defense of $\left(4^{*}\right)$ and $\left(4^{* *}\right)$.

\footnotetext{
${ }^{13}$ GPSP is supposed to follow from a broader recombination principle. See $₫ 4$ for discussion.
} 


\subsection{Premise (4)}

Both conditional premises can be justified, in brief, as follows. Schaffer's monism commits us to the existence of a single fundamental substance, and, further identifies this substance with spacetime. If there are possible worlds that are island universes, then there are worlds at which more than one spacetime exists. No such world is monistic - what is basic (spacetime) at such a world is many; it is not one. Moreover, this is true irrespective of whether the worlds at issue are weak island universes or strong island universes: in both cases we have two disconnected spacetimes and so, it would seem, two fundamental substances.

There are, of course, various ways to resist both the modest and proud arguments against monism. In both cases, there are two places where Schaffer might take a stand. First, accept the possibility of island universes, but deny that the possibility of island universes undercuts monism. Second, deny the possibility of island universes. We consider the first response in $\$ 3$ and the second response in $\$ 4$. Before that, we consider (and reject) one way in which Schaffer might deny that the possibility of island universes undercuts monism.

The way is just this: monism is true, but not necessarily true. monism becomes a contingent thesis. This deals with the objection from the islands of plurality; the possibly of pluralism is no threat to a form of monism that is merely contingently true.

We think that this form of monism is coherent. But we are uncertain as to whether or not anyone should adopt it, for at the moment the arguments in its favour seem substantially underpowered. After all, the gunk argument will no longer serve to motivate contingent monism; merely that gunk is possible does nothing to undermine pluralism if pluralism and monism are not necessarily true. Similarly, the truthmaker argument will not survive if monism is merely contingent (if objects other than the monistic substance are compossible with it, then the existence of the monistic substance cannot necessitate the non-existence of other objects). Of course, there are other arguments in monism's favour, as we indicated. But these arguments have been robustly challenged (e.g. Lowe (2012), Morganti (2009)). Perhaps there is enough left in the dialectical tank to fuel the motivations for monism, but we are doubtful. At the very least, we think that the monist ought to do much more to motivate contingent monism as a thesis.

\section{Schafftermath}

The first, and most straightforward response, to the proud argument proceeds as follows. At island universe worlds the monistic substance is a mereological fusion of spacetimes. The disconnected spacetimes are both individual concrete objects, the mereological sum of which is metaphysically prior to its parts: the mereological sum of the spacetimes is ontologically prior to the spacetimes themselves. Note that mereological fusion is not the only option for drawing the two spacetimes together. We might, instead, appeal to a primitive 'world-mate' relation, contending that the two island spacetimes, though spatiotemporally disconnected, find themselves in the same world, and so are world-mate-related. In general, there will be a number of such relations that might play a similar role. All of them would be abundant - and not sparse - relations, and so would preserve the fact that these are island universes we are considering.

In outline, our worry with this response is that Schaffer's account of metaphysical dependence makes this worldmate picture and its ilk implausible. According to Schaffer — and as we saw in \$1.2-integrated wholes are prior to their parts; non-integrated parts are prior to their sums. Island universes are paradigm case of non-integration, for we have non-spatio-temporally-connnected spacetimes. There is no spatio-temporal connection (or, so far as we can see, any other connection sufficient for integration) between the spacetimes. The two spacetimes are, as it were, more like a pile of rocks than like an integrated organism. That being the case, the spacetimes are ontologically prior to their mereological sum. By Schaffer's own lights, this island universe is not monistic. By Schaffer's own lights, then, monism fails.

This is only the outline of a response. Much more detail is required. Thus: in $\$ 1.2$, we noted that there is more to the importance of integration than common sense alone. Integration plays a crucial role in Schaffer's nomic argument for Spinozian Monism. On the one hand, it is partly definitional for Schaffer of what it is to be a substance that it displays integration. On the other hand, we can use the fundamental laws to test for the presence/absence of integration and thus as an important test for substance-hood.

As a consequence, our argument needs to be put in terms of nomic integrity. Thus: according to Schaffer, an entity is a substance just when it evolves according to the fundamental laws. In order for the fusion of two island universes to be a substance, then, it should follow that the fusion evolves according to a single set of fundamental laws; the fusion must 'act as one'. Given the role that nomic integrity plays in defining 
substancehood, whether or not the argument from island universes is a successful argument against Schaffer's monism depends upon whether we are considering weak island universes or strong island universes.

With respect to weak island universes: in the example involving Crowell solutions to the Wheeler-DeWitt equation, a case may be made that the two island universes evolve according to a single set of fundamental laws, and thus that the fusion of the two universes possesses substancehood. The case is, after all, a single solution to the Wheeler-DeWitt equation. It wouldn't be crazy to think this is a candidate to be a fundamental law of quantum mechanics. Of course, the Wheeler-DeWitt equation on its own won't do: we will also need general relativity to describe the broad mechanics of each of the two spacetimes. But, together, relativity and quantum mechanics do seem to be laws under which the case can be described as evolving in Schaffer's sense.

It may then seem that the weak island universe case - whereby a wormhole connects two spacetime cosmologies with some probability - is one that satisfies the nomic test for integration, and thus as a case that may ultimately be compatible with Schaffer's monism. Indeed, looked at in this light, the case is similar to one that Schaffer (2013, p. 83) considers:

What about a cosmos that contains closed subsystems? For instance, consider a 'two-monad' world of two non-interacting windowless bubbles, with purely inertial laws.

Of this case, Schaffer is careful to point out that "the whole system evolves by the fundamental laws, but so does each monad." So too, in the weak island universe case imagined, Schaffer has the option of treating the whole system of two island universes probabilistically related by a quantum event of tunneling as a single system evolving according to fundamental laws.

We are not totally convinced. We are uncertain as to whether all of the terms integral to Schaffer's account of dependence can be made sense of with respect to even weak island universes. For instance:

State The state of a thing at a time is its fundamental intrinsic character at that time.

Prediction The prediction for a state is the temporal evolution that the fundamental laws output on the basis of the state.

Behavior The behavior of a thing is the temporal evolution it actually displays.

Each of these notions is temporal. There is no overarching temporal structure in which to embed the two spacetimes under consideration such that they can be said to have $a$ fundamental intrinsic character at that time; nor is it easy to see how, in the absence of an overarching temporal structure we are to speak of 'temporal evolution' of any given laws. Thus, we are suspicious. But perhaps sense can be made of this temporal talk. In that case, we would do well to focus our efforts on strong island universes.

In the case of strong island universes, our appeal to nomic conceptions of integration gets good traction and we develop a clear reason to reject monism. As discussed above, a strong island universe can be generated by taking a single spacetime manifold $M$ and dividing it into two individuals $I_{1}$ and $I_{2}$, and then applying GPSP to the fusion $S$ of those two individuals to get to a world in which $S$ exists on its own, absent the spatiotemporal relations between $I_{1}$ and $I_{2}$ in $M$. Now, let's consider such a world a bit more carefully, and from the perspective of the fundamental laws. With respect to spacetime, the fundamental law at issue is the Einstein field equations:

$$
R_{\mu \nu}-\frac{1}{2} R g_{\mu \nu}=\frac{8 \pi G}{c^{4}} T_{\mu \nu}
$$

The important part of the field equations for present purposes is the stress-energy tensor, $T_{\mu \nu}$. Very roughly, the curvature of a spacetime is a function of the distribution of mass and energy throughout spacetime, which is partly encoded by $T_{\mu \nu}$. When we 'cut' a manifold in two via the application of GPSP, we are left with two spacetimes that have different distributions of mass and energy, resulting in different values for $T_{\mu \nu}$. The upshot: the two island spacetimes will have different geometric structures. This will have a range of important implications, the most crucial of which being that gravity will behave differently in the two spatiotemporal manifolds. In essence, then, two manifolds produced by a GPSP cut will correspond to different solutions to the Einstein field equations. This, in turn, will result in completely different behavior for the two universes. One manifold might, for instance, contain a lot of mass-energy and thus be highly curved; the other may contain very little mass-energy and thus be basically flat. 
What's more, there just isn't a fundamental law that we know of according to which both island universes evolve. It certainly won't be the field equations; the field equations cannot handle disconnected spacetimes. At best, the equations can tell you the curvature of one or more connected spaces; it can tell you about the curvature of each island universe individually. But the equations won't yield a solution for the two disconnected spacetimes.

(To illustrate, albeit somewhat metaphorically: imagine you have a piece of paper and you hold one edge, whilst bending the other around. Because all of the points that constitute the piece of paper are connected, the entire sheet is subject to an overall curvature, a curvature that can be neatly described by a single function. But suppose you take that same sheet and cut it in half and then bend each half individually in different ways. You will need two distinct functions to handle the two distinct instances of curvature and while there may be a function that can handle both, it won't be either of the initial functions. So too in the case of relativity: a GPSP cut results in two solutions to the field equation. The same field equation cannot then be used to describe both manifolds at once. Some further equation might do the trick, but we have no idea what it is or whether it is a candidate to be a fundamental law. ${ }^{14}$

To press the point about integration a bit further, consider this: a GPSP cut need not produce two island spacetimes, both of which are describable by solutions to the field equation. To see this, begin with a manifold $M$ which has a localized region, $\mathrm{R}$, that is completely flat, but the rest is curved. Now take that region and its complement, $R^{*}$, and perform a GPSP cut. The result will be (i) one spacetime, $R$, that may be describable by Newtonian mechanics, since there won't be any spacetime curvature to produce the exceptions to Newton's gravitational theory that motivate general relativity and (ii) one spacetime, $R^{*}$, that is curved and thus requires Einstein's field equation to get gravitation right. In this situation, we have no hope whatsoever of somehow 'unifying' the behavior of $R$ and $R^{*}$ under a single fundamental law that is not hopelessly gerrymandered. Indeed, once we see how to perform the kind of GPSP cut under consideration, a number of further examples arise: take any region $\mathrm{R}$ of a manifold $M$ that, on its own, is not a solution to the field equations. There are many such regions, because there are many manifolds that are not solutions to the field equations. So there are many island universes that won't obey anything like a global set of general relativistic principles.

Now, Schaffer says that if $x$ and $y$ merely co-evolve according to distinct laws $L_{1}$ and $L_{2}$, then $x$ and $y$ do not act as one. In such a circumstance, $x$ and $y$ would lack sufficient nomic integration to be considered a single substance. We accept this criteria. So $x$ and $y$ (in this case, our distinct spacetimes) must evolve according to some fundamental law, $L_{3}$, in order to act as one and to count as a single substance. In the case just described, there is no obvious candidate for playing the role of $L_{3}$ and so we have no nomic integration and no monistic substance comprised of disconnected spacetimes.

\subsection{Response to strong islands: there's always a law}

Schaffer may respond: in all putative island universes we might simply suppose that there is always a fundamental law, $L_{3}$, available such that spacetimes $x$ and $y$ evolve according to $L_{3} ; L_{3}$ will simply be the conjunction of $L_{1}$ and $L_{2}$. For two reasons, we don't think that this option is live for Schaffer. First, in perfectly general terms, it cannot be the case that, for any two laws, their conjunction is itself a law. The conjunction of quantum theory and the special theory of relativity, for instance, do not form a single unified law-though both of them are law-like. More is required of $L_{3}$ 's being a law than it merely be the conjunction of two other laws. Whilst we concede that we cannot provide exhaustive criteria as to when two laws, $L_{1}$ and $L_{2}$, can be subsumed into a more fundamental law, $L_{3}$, we are nonetheless confident that merely generating a conjunction out of $L_{1}$ and

\footnotetext{
14 The possible scenario we are aiming to establish is one in which there are two disconnected spacetimes. We get to that possibility by 'cutting up' a spatiotemporal manifold. There is no guarantee, however, that cutting a spatiotemporal manifold in two will yield two manifolds, each of which has a metric structure permitted by general relativity. To show that there is a possible scenario generated by a GPSP cut that is compossible with the laws of the original scenario would take us too far afield (though we believe it to be a mathematically tractable outcome of the field equations). For now it is enough to emphasise that the result of a GPSP cut need not be compossible with the actual laws. So long as the result of the cut is two entities, $E_{1}$ and $E_{2}$, such that $E_{1}$ and $E_{2}$ are not externally unified, we have the result we seek. That said, there is a gap in our argument here that Schaffer may exploit. If it could be shown that the possible scenario produced by a GPS cut is not compossible with the actual laws, it is at least an open question that there is some global law in that world by which the two entities produced by the cut 'act as one'. If pressure were exerted on this part of our argument, we would defer to the argument in \$3.3, which shows how to produce a pluralist world using GPSP regardless of what's going on with the laws.
} 
$L_{2}$ will not do the job. Second, we think that Schaffer himself must resist the claim that the mere conjunction of $L_{1}$ and $L_{2}$ generates a new law, $L_{3}$. if he doesn't resist, then it will be very difficult (impossible?) to draw a distinction between 'evolving as one' and 'co-evolving' - a distinction that must be drawn if Schaffer is to differentiate his monism from pluralism. Here is our concern in more detail. We, following Schaffer (2013), think that it's possible to at least describe a pluralistic world. So, consider a description of a genuinely pluralistic world, where there are two fundamental laws of physics, $L_{1}$ and $L_{2}$, and two distinct mereologically complex concrete objects, $C_{1}$ and $C_{2}$. $C_{1}$ evolves according to $L_{1} ; C_{2}$ evolves according to $L_{2}$. If we said that, for any $L_{1}$ and $L_{2}$, there is a conjunction, $L_{3}$, such that $L_{3}$ is a fundamental law, then it will follow that this putatively pluralistic world is in fact monistic: in all such cases the mereological fusion of $C_{1}$ and $C_{2}$ will evolve according to $L_{3}$. Thus, even our best efforts to describe a pluralistic world fail. It then turns out to be analytic that monism is true. But monism is supposed to be a substantial metaphysical thesis, to be argued for via a variety of a priori and a posteriori arguments (see above). It is not supposed to be a trivial consequence of the fact that we can conjoin statements of seemingly independent physical laws. In fact, we will go slightly further. If Schaffer took the view that merely conjoining statements of laws showed monism to be true, we would take this as a reductio of monism itself. Of course we can conjoin independent clauses of natural language. No metaphysical theses should be derived from that trivial semantic fact.

\subsection{Response to strong islands: Reason $X$}

First, a quick bit of pre-amble:

(1) We say that two disconnected spacetimes do not act as one according to any actual law.

(2) Schaffer responds: there is always some non-actual law governing the two spacetimes, and it is the conjunction of the laws that govern each spacetime individually.

(3) We respond: the conjunction of the two laws is not a law; the (important) contrast with pluralism is lost.

Here, then, is the imagined response:

(4) There is always some non-actual law governing the two spacetimes for reason X.

We have no idea what reason $\mathrm{X}$ is. Nonetheless, someone might say that the burden of proof is on us to rule out the existence of reason X. Can we say anything on this score? We think that we can.

(5) Consider an island universe, $W$, in which the two spacetimes, $S T_{1}$ and $S T_{2}$, are described by distinct solutions to the field equations; $S_{1}$ and $S_{2}$, respectively.

(6) The field equations say everything there is to say, nomically, about a spacetime.

(7) According to Schaffer, there must be a further law, $L$, such that $L$ governs $S T_{1}$ and $S T_{2}$.

(8) $S_{1}$ and $S_{2}$ say everything there is to say about spacetimes $S T_{1}$ and $S T_{2}$, where $S T_{1}$ and $S T_{2}$ exhaust world $W$ (nothing exists at $W$ that isn't at either $S T_{1}, S T_{2}$ or their mereological fusion); so, $L$ won't say anything about the nomic structure of $W$ that isn't already said by $S_{1}$ and $S_{2}$.

(9) We should not posit laws in worlds where those laws say nothing about the nomic structure of that world that isn't already said by other laws. (What would be the point of such a law? What would it $d o$ ?)

(10) We should not think that $L$ exists at $W$.

In brief: because the additional law to be posited, $L$, simply won't make any nomic contribution to $W$, that isn't already made by the solutions to the field equations $S_{1}$ and $S_{2}$, so we shouldn't posit $L$. To resist the argument, one would need to show that there's something that the field equations for two disconnected spacetimes cannot capture, nomically speaking. Perhaps this can be shown, but we are doubtful. That's because the nature of a spacetime just is whatever solution is yielded by the field equations. A spacetime does not come apart from its associated solution, and is completely described by that solution. To deny this is to misunderstand the importance of the field equations for explicating the nature of spacetime. Thus, even this merely imagined reply is to be rejected. 
And so it is that the Schafferian gambit under consideration, of treating the 'fusion' of two spacetimes as a fundamental substance, falls short in the case of strong island universes. The problem is simply that there is no sense in which the fusion 'acts as one'. Strong island universes fail Schaffer's nomic test for substancehood. 15

\subsection{Response to strong islands: give up integration}

At this point, one might be tempted to respond as follows. All that the arguments marshalled against monism so far have shown is that monists cannot accept the argument from integrity for monism, and nor can they accept the nomic integration of substances. This still does not get to the core of monism, however. For a different kind of monist, one who gives up the nomic test for substancehood, who motivates their view without integrity (of the substance, that is), the possibility of strong island universes can be accepted without trouble. Such a monist maintains that the one fundamental entity is always the most inclusive fusion - the MAXFUS, but it need not act as one. Accordingly, in our world, the one fundamental substance is the spatiotemporal manifold, since that is the MAXFUS in our world. In a strong island universe with $n$ spacetimes, the one fundamental substance is the MAXFUS of those spacetimes. And so it goes.

Call this position: MAXFUS monism. There are two difficulties with a position of this kind. First, while integration may be a negotiable commitment, it is an important commitment; else, what are the criteria for unity deployed by a monist? The monist claims that common sense recognises cases of parts being dependent upon wholes (and vice versa). Island universes look to be cases of the whole being dependent upon parts. The monist claims that integration from physics is a clear signal of unity. Island universes are a case where there is no transspace-time unity. Thus, this looks to be a clear case where we have a plurality. Lastly, the monist has some things to say about emergence. Schaffer (2010: 50-57) argues: the cosmos can have various emergent features (quantum entanglement being one example); the cosmos can only have these features if there is a single entity (rather than a plurality of entities) that instantiates these features; therefore, the actual world is monistic. But it seems to us that there is no obvious emergent property of a MAXFUS monism. Rather, the intrinsic nature of the maximal fusion is fixed by the natures of the spacetimes. That being the case, we think that considerations of emergence would lead us to conclude that a fusion of the sort described need not be monistic at all. So this looks to be a case where the nature of the whole is determined by the nature of the parts-or at least to be neutral.

Note, then, that by every criteria the monist gives us, we should either avoid a commitment either way (in the case of emergence) or else reject the claim that an island universe is a monist universe. It's hard to see the unity of these claims as being a negotiable commitment of monism. These are all of the criteria that the monist has to deploy in an argument for monism.

The second problem with MAXFUS monism is a rather general problem with the strategy under consideration. The basic strategy deployed by the MAXFUS monist is this. For any world in which there are two island spacetimes, those two spacetimes are grounded in a single fundamental entity E. For the MAXFUS monist, this single fundamental entity is the maximal fusion, but it need not be. It could be anything, so long as there is just one thing $E$ such that all grounding relations terminate in $E$. The trouble is that GPSP can be used to directly produce worlds in which there are at least two grounding chains that do not terminate in a common element. To see the basic idea, consider Bricker's account of how to produce an island universe:

To get from (GPSP) to the possibility of island universes, one can start with any world containing two or more individuals and strip off relations, that is, polyadic universals or tropes. One way: apply (GPSP) to the world minus all polyadic universals or tropes. In the possibility that results, distinct individuals are absolutely isolated universes [i.e. strong island universes]. Another way is more selective, but works only for tropes: divide the world into two distinct individuals and apply (GPSP) to the world minus all polyadic tropes connecting the two individuals. In the possibility that results, the duplicates of the two individuals are absolutely isolated universes. (Bricker 2001: 38)

\footnotetext{
15 This result follows even once we have conceded the relatively liberal account of what it is for a world to be unified offered above. For while these island universes are certainly unified via a non-spatiotemporal relation, the candidate entity is not unified by the kind of relation that features in a fundamental law; at least not one of which we are presently aware.
} 
GPSP is powerful indeed. The trouble for the MAXFUS monist is that GPSP allows us to strip away grounding relations, as much as any other kind. This is easiest to see in the first, rather extreme, case that Bricker outlines (though we modify it to take into account the fact that the world is a MAXFUS world).

Take a MAXFUS monist world, $W_{1}$. In that world, there are two island spacetimes $S T_{1}$ and $S T_{2}$ that are both grounded in the maximal fusion. Now take a sub-region of spacetime from $S T_{1}, I_{1}$, which will be one of $S T_{1}$ 's proper parts. $I_{1}$ itself grounds a range of further parts, $p_{1} \ldots p_{n}$. Next take a sub-region of spacetime from $S T_{2}, I_{2}$, which will be one of $S T_{2}$ 's proper parts. $I_{2}$ itself grounds a range of further parts, $p^{*}{ }_{1} \ldots p^{*}$. Now, note that each of the $p_{n}$ in a MAXFUS world stands in the relation of having a common ground with each of the $p^{*}$, which is to say that all parts share the MAXFUS as the common ultimate basis. Now, take the two individuals $I_{1}$ and $I_{2}$ and, as GPSP allows, start stripping off relations to get to a world, $W_{2}$. Here is the (somewhat metaphorical) recipe to construct $W_{2}$. A visual representation of the recipe follows in Figure 1.

First, strip off all spatiotemporal relations connecting $I_{1}$ to the broader $S T_{1}$ and then get rid of the remaining chunk of spacetime as well, the chunk that is $I_{i}$ 's complement. Then do the same for $I_{2}$. Next, strip away the relation of having a common ground that obtains between $I_{1}$ (and any part thereof) and $I_{2}$ (and any part thereof). Now look at what you've made. You have made a world in which none of $I_{i}$ 's parts share a common ground with any of $I_{2}$ 's parts. Accordingly, $I_{1}$ cannot be grounded in $I_{2}$ or vice versa. Rather, there must be two distinct chains of grounding, one leading down from $I_{1}$ 's parts to terminate in an element $E$ (probably $I_{1}$ itself), and another chain leading down from $I_{2}$ 's parts to terminate in an element $E^{*}$ (probably $I_{2}$ itself) such that $E$ is distinct to $E^{*}$.

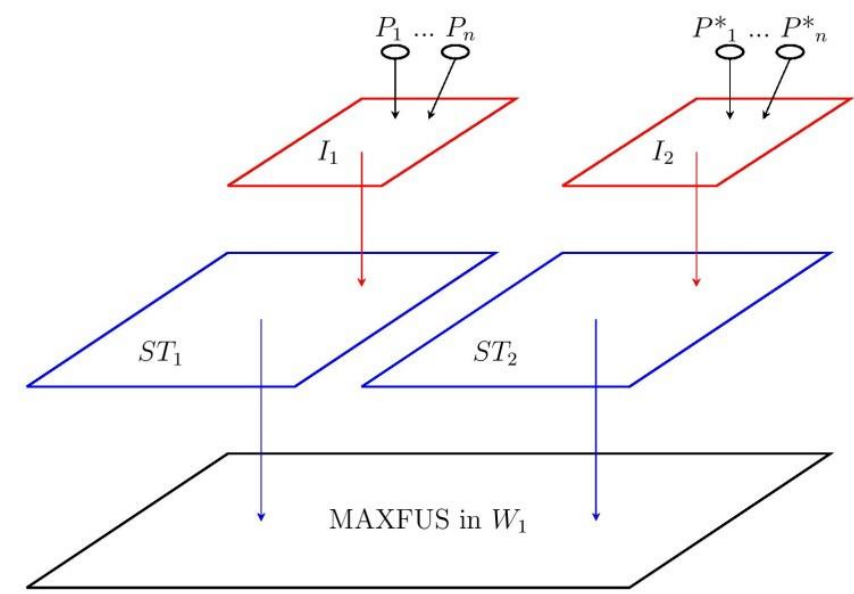

World $1\left(W_{1}\right)$

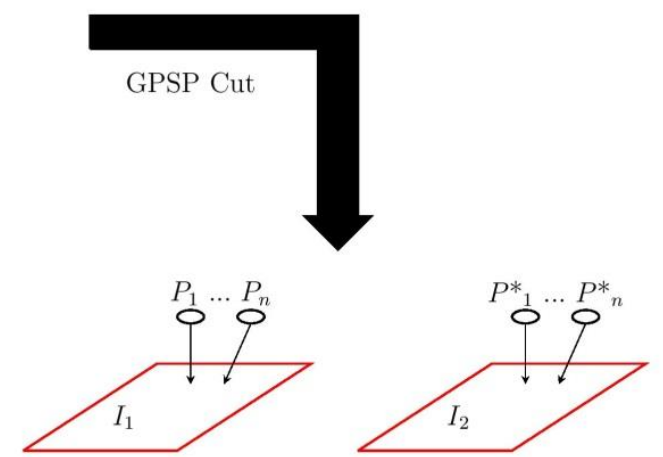

World $2\left(W_{2}\right)$

Figure 1: GPSP cut to remove the relation of 'having a common ground'.

The upshot? $W_{2}$ is not a monist world; it is a pluralist world (see Figure 1). So possibly, monism is false. So monism is false.

A note about this demonstration: it is quite general, in two important ways. First, we have focused on MAXFUS monism. Nonetheless, the same line of reasoning will apply to any entity $E$ that is used to ground two spacetimes in an island universe so as to render such a thing compatible with monism. In each case, there will be a GPSP cut available that may be used to strip away relations of having a common ground, resulting in a world with two chains of grounding that fail to terminate in a common element. Second, we have focused on disconnected spacetimes, and certain relations of having a common ground that obtain between their parts. But the focus on spacetimes is, really, incidental. Exactly the same argument will succeed for any two entities, whether they are spacetimes or something else entirely. This is important as it shows that nothing about our argument hangs on the identification of objects with regions of spacetime. Let that assumption go and the argument we have just outlined will go through just the same. The difficulty, in a nutshell, is that GPSP strips away globally unifying grounding relations as much as any other kind, and so it won't help the monist to try and unify island universes under the grounding relation, and yet they clearly must if monism is to remain intact. For a 
world that is not unified under the grounding relation just is a world with a plurality of fundamentals. So that leaves just one line of resistance open to the monist: reject GPSP.

\section{Recombination}

GPSP is a strong principle of recombination. It allows free recombination of properties and relations, in addition to particulars. For all we have said, the monist is not compelled to adopt this particular recombination principle and so it may be argued that all we have revealed is a tension between monism and a particularly strong recombination principle. So much the worse for GPSP, says the monist, who then departs, monism intact.

In rejecting GPSP, however, something must be put in its place. A recombination principle of some kind is needed to produce the plurality of worlds. Moreover, in the current context the relevant principle must be one that forecloses the possibility of island universes. We cannot hope to rule it out that there is some such recombination principle available. Instead, we will outline two difficulties for the monist who proceeds along these lines. The first difficulty lies in finding a principle of recombination fit for service.

Now, at first blush, this might not seem so difficult. For there is a well-known recombination principle that does not allow for island universes: Lewis's (1989: 87-92). Following Wilson (2015: 146), this principle may be stated as follows:

HD (L-Combinatorialism): Every occupant of spacetime existing at any actual or possible world is such that one of its duplicates (sharing all Hume-intrinsic properties) can coexist with one of the duplicate(s) of any non-overlapping occupant(s) of spacetime existing at any actual or possible world(s), or fail to coexist with one of the duplicate(s) of any occupant(s) of spacetime existing at any actual or possible $\operatorname{world}(\mathrm{s}) .{ }^{16}$

As Wilson argues, however, this principle of recombination is inadequate. One of the central difficulties with the principle is that it rules out genuine scientific possibilities. That's because the principle permits the recombination of entities that occupy spacetime only. By restricting recombination to entities within spacetime, spacetime is effectively held fixed as the background framework for possibility. This rules out any possibilities that are not spatiotemporal in nature. As Wilson (2015: 153-154) puts the point:

... a comparatively vast array of possibilities - all those associated with spaces in some or other respects different from ordinary spatiotemporal space - are simply left out of Lewis's account. Lewis might try to maintain that these possibilities are not genuine, but in doing so he would be on shaky ground, by his own lights, for he counsels us to look to the sciences to get a handle on what is fundamental (or "perfectly natural"), and it is the sciences that have raised to salience the possibility of non-spatiotemporal spaces.

For instance, theories according to which the background framework in which entities exist is a configuration space are flatly ruled out by Lewis's principle, even though (as mentioned previously) these are possibilities that are taken seriously by science. ${ }^{17}$ So Lewis's principle won't do. The trouble is that in order to modify the principle so as to rope in those cases that are treated as live possibilities it seems we are forced to expand the principle so as to allow for free recombination of relations, bringing it into line with GPSP. For instance, in order to be able to take a configuration space account of the actual world seriously, we must be able to accept, as a possibility, a world that has the same occupants as the actual world, but the underlying relational framework unifying those occupants is completely different (the relations would probably be based on similarity or some kind of lawful unity such as provided by the Wheeler-DeWitt equation, rather than spatiotemporal relations). Now, as Wilson notes, there are certainly recombination principles available that allow for free recombination on relations. Wilson identifies, as an example, Armstrong's principle, according to which the plurality of worlds is created by free recombination of the actual universals. The difficulty, however, with any combinatorial principle that operates at the level of relations, is that it seems to open up the possibility of island universes. That's

\footnotetext{
16 A similar account of Lewis's principle of recombination is offered by Effird and Stoneham (2008, p. 489):

For any sequence of intrinsically distinct objects $x_{1}, x_{2}, x_{3}, \ldots, x_{m}$ and any sequence of cardinals $\left(n_{i} \geq 0\right) n_{1}, n_{2}, n_{3}$, $\ldots, n_{m}$ and any spatiotemporal relation between those objects, there exists a possible world that contains: exactly $n_{1}$ duplicates of $x_{1}$, exactly $n_{2}$ duplicates of $x_{2}$, exactly $n_{3}$ duplicates of $x_{3}, \ldots$, exactly $n_{m}$ duplicates of $x_{m}$, in that spatiotemporal relation.

${ }^{17}$ For an excellent discussion of the metaphysical implications of configuration space, see Paul (2012).
} 
because, as we saw with GPSP, it is the ability to strip away and thus recombine relational facts that enables one to derive universes of this kind.

The first difficulty for the monist, then, is to provide a principle of recombination that does better than Lewis's, in that it gets the space of scientific possibilities right, without being so liberal as to allow for island universes. Perhaps such a principle can be developed, but we are not sanguine. After all, the weak island universe case considered above (with two spacetimes bridged with some probability) just is a scientific possibility. So that case, at least, must be permitted. The second difficulty is this: even if such a principle of recombination could be developed and used to successfully derive the plurality of worlds, it would be potentially costly. Any such principle of recombination would need to rule out failures of external unification. As above, we continue to assume that two parts of a world $W, P_{1}$ and $P_{2}$ are strongly separated just when $P_{1}$ and $P_{2}$ are non-overlapping and no part of $P_{1}$ stands in any sparse external relation to $P_{2}$ and thus that a world $W$ is externally unified by a sparse external relation when it is not the sum of two parts that are strongly separated. Accordingly, the recombination principle needed by the monist must be subject to the following exclusion principle:

Forced External Unification (FEU): For any $x$ and any $y, x$ and $y$ are related by some sparse external relation $R$ at every world $W$.

FEU requires the existence of necessary connections. In particular, it requires that necessarily, each $x$ is connected to each $y$. Note that the necessary connection is not one of requiring each $x$ and each $y$ to be related by the same R-relation. It is, rather, the requirement that there be always be some connection or other between any two entities forged by an external relation, or else a world won't be externally unified.

There are then two problems with FEU. The first (perhaps less pressing) relates to Hume's Dictum: ${ }^{18}$ that there are no necessary connections between distinct existences. FEU flies in the face of that dictum. The intuitive pull of the dictum constitutes a basis for resisting any recombination principle that implies FEU. Now, one might argue that invoking Hume's Dictum is not dialectically effective against the monist. As previously discussed, the monist maintains that the substance acts as one. If laws are tethered to substancehood in this fashion, then one may well be forced to accept fundamental laws: laws that do not supervene on the Humean mosaic. Accordingly, the monist is already committed to a non-Humean metaphysics. So they should not find the proposed motivation for denying FEU compelling.

But we must be careful. In accepting fundamental laws one would, presumably, be opting for an account of laws along the lines developed by Armstrong (1983), according to which laws of nature are necessitation relations between universals. It can be quite tempting, then, to think that such an account of the laws of nature flatly contradicts Hume's Dictum. Looking toward Armstrong's broader metaphysics, however, it is clear that this is not so. Armstrong $(1989,2004)$ accepts a combinatorial account of possibility that is designed to fit within the scope of that Dictum. For Armstrong, the plurality of worlds is derived via recombination of the actual universals, with no exclusions and no necessary connections. Indeed, Armstrong goes to great lengths to explain away the appearance of any absolutely necessary connections between universals (whether he is successful or not, is another matter entirely, see Lewis (1992)). What, then, can it mean to say that universals stand in necessitation relations to one another? What this means is that the universals physically but not absolutely necessitate one another. Since it is absolute necessitation that is targeted by Hume's dictum, a non-Humean account of the laws is compatible with a Humean approach to possibility. So the monist is not antecedently committed to rejecting Hume's Dictum, at least not because they invoke fundamental laws. It is, then, a cost of some order for them to accept FEU.

The second (perhaps more pressing) reason to worry about FEU is that it seems to commit one to entities that do some excluding. For it follows from FEU that:

\footnotetext{
${ }^{18}$ We are reluctant to put too much weight on the preservation of Hume's dictum, given the concentrated attack on that dictum offered by Wilson (2010). That said, we believe that our opponents, who tend to be Humeans, will wish to preserve the dictum, and so it is a cost of some order to them that the dictum must be given up (though of course they already have their work cut out for them if Wilson is correct and the dictum is indefensible).
} 
Spacetime Exclusion (STE): For every world $W$ with spacetime $S$ there is no further spacetime $S^{*}$ such that $S^{*}$ is strongly separated from $S$.

If STE is false, then so is FEU. ${ }^{19}$

STE is a troubling principle, however. STE suggest that a spacetime is an entity such that, if it exists, an entity of a particular sort does not exist. We're familiar with such entities. They are close cousins of Armstrong's totality fact. Armstrong's totality fact is a second order fact that sums the first order facts and says of them: no facts other than these facts exist (and 'these' is taken to include reference to itself). Given STE, it now seems that the actual spacetime is an excluder. It says, of itself, that no spacetime strongly separate from this one exists. That's pretty close to the 'that's all folks' nature of a totality fact. Rightly, the totality fact has a bad reputation. As we saw in $\$ 1.4$, we typically reject such 'negative' entities, such is the bad reputation bestowed by endorsing negative ontology. Given that STE would similarly threaten the good name of monism, we think there is further reason to doubt FEU. ${ }^{20}$ And note that the problem lies not just with STE. FEU commits one to a range of principles like STE. Each spacetime must not only exclude the existence of a strongly separate spacetime, it must exclude the existence of a strongly separate anything. Indeed, everything must exclude a strongly separate anything in this sense. Suddenly there is a great deal of excluding work going on and thus a fair amount of potentially suspect ontology.

Of course, the monist may just wear the cost of FEU. But we believe there is at least a challenge that must be answered. And that challenge lies in a surprising place for the monist: they owe us an account of recombination that does not yield island universes, one that may be independently motivated. With such an account in hand, they can reasonably set island universes aside. Until then, however, they have reason to worry.

\subsection{A Final Response}

At this point the monist may insist that monism is necessarily true and simply engage in a dogmatic foot-stamp in the face of the argument from the possibility of island universes. To refine the view as best we can, this dogmatic spacetime monist holds that it is necessarily true that each possible world consist of a single spacetime. Perhaps they concede it to be a vice of their view that there are no island universes, but in the end, they suggest, the pay-off is worth bearing this vice. And would matters really be so bad? After all wouldn't this just put Schaffer in the same boat as the modal realist, who (following Lewis) treats worlds as maximal spatio-temporal sums?

We believe that it does, indeed, put the monist in the same boat as the Lewisian modal realist. But we maintain, with Bricker, that the possibility of island universes is decisive against a Lewisian modal realist, and so it is decisive against monism as well. That being said, we do not believe the possibility of island universes ruins any version of modal realism whatsoever. As Bricker has also argued, a non-Lewisian modal realist can allow for the possibility of island universes simply by allowing plural quantification over worlds (2001: section 3.4). It seems, then, that it is really the monist who finds themselves all at sea when it comes to island universes, and their plurality.

\section{REFERENCES:}

Armstrong, D. 1983. What is a Law of Nature? Cambridge: Cambridge University Press.

Armstrong, D. 1989. A Combinatorial Theory of Possibility. Cambridge: Cambridge University Press.

Armstrong, D. 2004. Truth and Trutbmakers. Cambridge: Cambridge University Press.

Barbour, J. 1999. The End of Time. Oxford; New York: Oxford University Press.

Baron, S. and Miller, K. 2015. 'What is Temporal Error Theory?', Philosopbical Studies, 172(2), 2427-2444.

\footnotetext{
${ }^{19}$ To see this, note that for it to be true that, for any $x$ and any $y, x$ and $y$ are related by some external relation $R$ at every world $W$, every spacetime, $S$, must be such that there is no other spacetime $S_{n}$ (if there were such a thing, then there would be a spacetime, $S_{n}$, that is not related to spacetime $S$ - thus violating FEU).

${ }^{20}$ We also note that endorsing them would undercut Schaffer's claim to solving the problem of negative existentials without positing negative ontology; something that would further challenge the motivations for monism
} 
Barker, S. and Jago, M. 2012. 'Being Positive About Negative Facts', Philosophy and Phenomenological Research, 85(1), $117-138$.

Bricker, P. 1993. 'The Fabric of Space: Intrinsic Vs. Extrinsic Distance Relations,' Midwest Studies in Pbilosopby 18, French, Uehling, and Wettstein (eds.), University of Notre Dame Press, 271-294.

Bricker, P. 1996. 'Isolation and Unification: The Realist Analysis of Possible Worlds', Philosophical Studies, 84(2), $225-238$.

Bricker, P. 2001. 'Island Universes and the Analysis of Modality', in G. Preyer and F. Siebelt, eds., Reality and Humean Supervenience: Essays on the Philosophy of David Lewis. Rowman and Littlefield, 27-56.

Dodd, J. 2007. 'Negative truths and truthmaker principles', Synthese, 156, 383-401.

Efird, D. and Stoneham, T. 2008. 'What is the Principle of Recombination?', dialectica, 64(4), 483-494.

Lewis, D. 1986. On the Plurality of Worlds. Oxford: Blackwell.

Lewis, D. 1992. 'Critical Notice', Australasian Journal of Philosophy, 70(2), 211-224.

Lowe, E. J. 2012. 'Against Monism', in Goff. P. ed., Spinoza on Monism London: Palgrave Macmillan, 92-122.

Molnar, G. 2000. 'Truthmakers for Negative Truths', Australasian Journal of Philosophy, 78(1), 72-86.

Morganti, M. 2009. ‘Ontological Priority, Fundamentality and Monism', Dialectica, 63(3), 271-88.

Paul, L. 2012. 'Building the World from its fundamental constituents', Philosophical Studies, 158(2), 221-256

Priest, G. 2001. 'Truth and contradiction', Philosophical Quarterly, 50(200), 305-319.

Schaffer, J. 2003. 'Is There a Fundamental Level?’ Nous, 37(3), 498-517.

Schaffer, J. 2009. 'Spacetime the One Substance', Philosophical Studies, 145(1), 131-48.

Schaffer, J. 2010a. 'Monism: the Priority of the Whole', Philosophical Review, 119(1), 31-76.

Schaffer, J. 2010b. 'The least discerning and most promiscuous truthmaker', Philosophical Quarterly, 60(239), 307324.

Schaffer, J. 2013. 'The Action of the Whole', Proceedings of the Aristotelian Society 87(1), 67-87.

Simons, P. 2008. 'Why the Negations of False Atomic Sentences are True', in T. de Mey and M. Keinänen, eds., Essays on Armstrong. Acta Philosophica Fennica 84, 15-36.

Tallant, J. 2010. 'There's no existent like 'no existence' like no existent I know', Philosophical Studies, 148(3), 387400.

Tallant, J. 2013. 'Problems of Parthood for Proponents of Priority', Analysis, 73(3), 423-38.

Weingard, R. 1976. 'On the Unity of Space', Philosophical Studies, 29(3), 15-20.

Wilson, J. 2010. 'What is Hume's Dictum, and Why Believe it?', Philosophy and Phenomenological Research, 80(3), 595-637.

Wilson, J. 2015. 'Hume's Dictum and Metaphysical Modality', in B. Loewer and J. Schaffer, eds., A Companion to David Lewis. Oxford: Wiley, 138-158. 